\title{
Attractor Landscapes and Information Processing by Convective Obstacle Flows
}

\author{
Stuart Bartlett ${ }^{1,2}$, Yuk L Yung ${ }^{1}$ \\ ${ }^{1}$ California Institute of Technology, Pasadena, United States \\ ${ }^{2}$ Earth Life Science Institute, Tokyo, Japan \\ sjb@gps.caltech.edu
}

\begin{abstract}
We present recent results concerning the attractor landscape, memory, hysteresis and computation that can emerge in simple convective obstacle flows. In these systems a single phase fluid is heated from below and cooled from above. Small obstacles (one or two) are placed on the horizontal mid plane of the system and extract some fraction of the fluid's horizontal or vertical momentum. Horizontal momentum sinks tend to attract convection plumes. Vertical momentum sinks are bistable; the obstacle will either align with a convection cell centre or convection plume depending on initial conditions and the history of the system. The resulting attractor landscape can be exploited to produce a single bit memory or even elementary Boolean logic.
\end{abstract}

In this work we use numerical simulations of 2D convection flows to illuminate the rich dynamical behaviours that thermal fluids can exhibit. Natural convection has been studied for centuries, but many aspects of this non-linear phenomenon are still a matter of active research (e.g., Ahlers et al., 2009; Manneville, 2006). In this study we explored the effects of small obstacles placed in a driven thermal fluid system. The boundary temperatures are held constant and adjusted such that the dimensionless driving force is also constant.

Obstacles placed in such flows cause a break in their horizontal translational symmetry. When such obstacles, or momentum sinks, extract only horizontal momentum, the flow aligns plumes (maxima of vertical momentum) with the sink, as shown in Fig. 1(a). A similar attractor occurs when both horizontal and vertical momentum are extracted, as illustrated in Fig. 1(b).

These two attractors are identical except for the fact that the total momentum sink (Fig. 1(b)) is removing a significant quantity of kinetic energy from the flow, whereas the horizontal sink (Fig. $1(a))$ is not extracting any energy since the flow

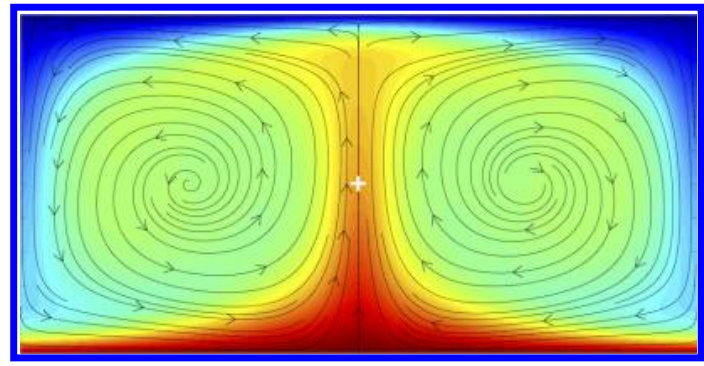

(a)

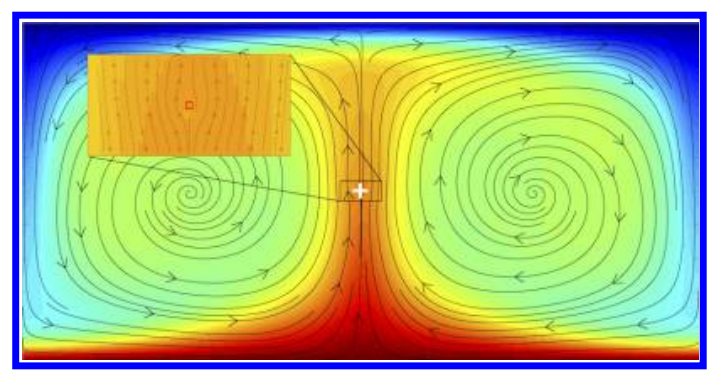

(b)

Figure 1: Typical flow structure of a convective fluid driven at a Rayleigh number of $R a=10^{5}$ in the presence of a small sink of momentum placed at $[x, y]=[W / 2, H / 2]$ (the white crosshairs show its location, its size is only $\sim 0.7 \%$ of the system height). a) Horizontal momentum extraction, b) Total momentum extraction.

field at that point (plume centre) is purely vertical (no horizontal momentum to extract).

When only vertical momentum is extracted by the sink, there are two types of attractor that occur: one in which either vortex centre aligns with the sink (Fig. 2(a)), and another in which either convection plume aligns with the sink (Fig. 2(b)). A detailed analysis of the fluid dynamical basis of these effects is given in Bartlett and Yung (2019).

The bistability and attractive/repulsive be- 


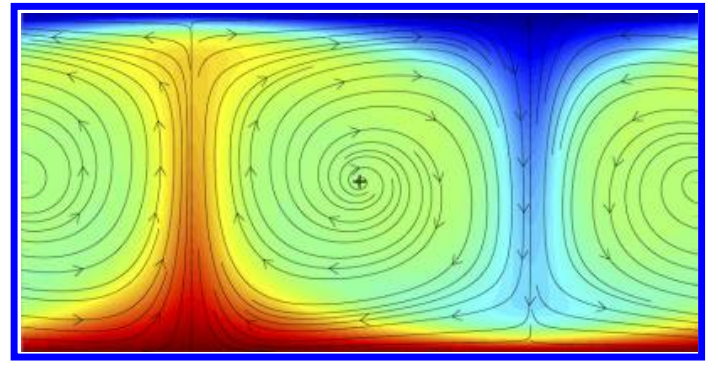

(a)

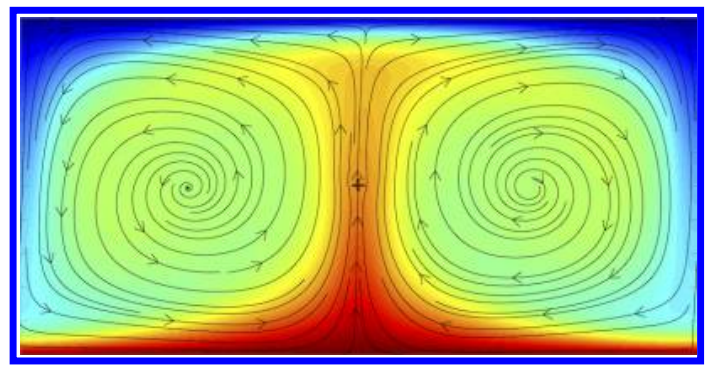

(b)

Figure 2: The two different types of attractor of a convective fluid driven at a Rayleigh number of $R a=10^{5}$ in the presence of a small sink of vertical momentum placed at $[x, y]=[W / 2, H / 2]$. a) Clockwise vortex attractor, b) Upwelling plume attractor.

haviour outlined above can be exploited to confer information storage and processing capabilities. Fig. 3 shows the history-dependence of the state of a flow system in which vertical momentum is extracted by the sink, but horizontal momentum extraction is switched on and then back off. Applying horizontal momentum extraction shifts the state of the system to the plume attractor, which is an absorbing state. Thus, when the parameters are returned to their original values, the system does not return to its original configuration. Convection flows can therefore store at least a single bit of information using this state history dependence.

Furthermore, simple Boolean logic, such as the OR gate, can be implemented using this simple system. In fact, several logic gates can be instantiated when two obstacles are placed in the flow Bartlett and Yung (view). These results suggest a new direction for the field of fluid computation (Adamatzky, 2018; Foster and Parker, 1970; Katsikis et al., 2015; Prakash and Gershenfeld, 2007), which has so far not explored the use of convection flows for simple computational logic.

We are now exploring the integration of convective logic gates such that entire computational

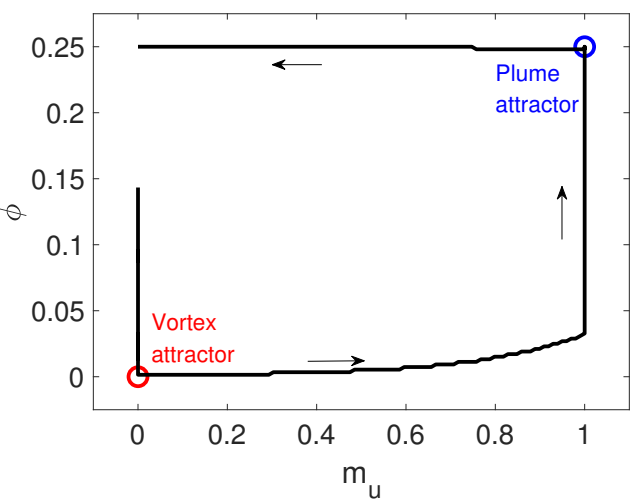

Figure 3: Phase space behaviour for a convective obstacle simulation with time-varying horizontal momentum extraction.

circuits can be constructed using only thermal fluid units. Results such as these suggest the use of information theory and computer science to understand a wide range of non-linear and nonequilibrium systems that traditionally lie outside of these fields (Feldman et al., 2008).

\section{References}

Adamatzky, A. (2018). The dry history of liquid computers. arXiv preprint arXiv:1811.09989.

Ahlers, G., Grossmann, S., and Lohse, D. (2009). Heat transfer and large scale dynamics in turbulent rayleigh-bénard convection. Rev. Mod. Phys., 81:503-537.

Bartlett, S. J. and Yung, Y. L. (2019). Convective flow in the presence of a small obstacle: Symmetry breaking, attractors, hysteresis, and information. Phys. Rev. E, 99:033103.

Bartlett, S. J. and Yung, Y. L. (Under review). Computation by convective obstacle flows.

Feldman, D. P., McTague, C. S., and Crutchfield, J. P. (2008). The organization of intrinsic computation: Complexity-entropy diagrams and the diversity of natural information processing. Chaos, 18(4):043106.

Foster, K. and Parker, G. (1970). Fluidics: components and circuits. Wiley-Interscience.

Katsikis, G., Cybulski, J. S., and Prakash, M. (2015). Synchronous universal droplet logic and control. Nature Physics, 11(7):588.

Manneville, P. (2006). Rayleigh-bénard convection: thirty years of experimental, theoretical, and modeling work. In Dynamics of Spatio-Temporal Cellular Structures, pages 41-65. Springer.

Prakash, M. and Gershenfeld, N. (2007). Microfluidic bubble logic. Science, 315(5813):832-835. 